\title{
Plane-polarized helicon waves
}

\author{
Francis F. Chen
}

Electrical Engineering Department

PPG- 1570

February, 1997 


\title{
Plane-polarized helicon waves
}

\author{
Francis F. Chen
}

Electrical Engineering Department, University of California, Los Angeles, CA 90095-1594

\begin{abstract}
By suitably adding right- and left-hand circularly polarized helicon waves in a cylinder, it is possible, in principle, to form linearly polarized modes, contrary to the notion that whistler waves must be circularly polarized. The planepolarized component is accompanied by a left-hand circularly polarized component which vanishes on axis but becomes important at large radii. The field lines of these two components and the energy deposition profile are computed for an illustrative case.
\end{abstract}

\section{INTRODUCTION}

Helicon wave sources are well known for producing high plasma densities suitable for use in materials processing [1]. Though helicon waves are basically whistler waves, and whistler waves traveling along a uniform magnetic field cannot be left-hand polarized, helicon waves can have either circular polarization [2]. This is because bounded whistlers behave differently from whistlers in an infinite plasma [3]. Since in most experiments a linearly polarized antenna is used-most often a Nagoya Type III antenna [4]—one might expect that the mode that is excited would be a superposition of the right- and left-handed circularly polarized modes, forming a plane-polarized wave [5]. Recently, Kim et al. [6] reported detecting such azimuthal standing waves in an experiment. These have also been seen by Ellingboe and Boswell [7], but in that experiment the standing waves were more prominent before the onset of helicon waves than after. The purpose of this paper is to show that plane-polarized helicons are possible in theory. For this purpose, we consider a uniform plasma without dissipation, but the effects of damping and plasma profiles will be discussed.

\section{EQUATIONS}

Consider an infinitely long plasma of uniform density $n_{0}$ filling a conducting or insulating cylinder of radius $a$ in a uniform, coaxial magnetic field $\mathbf{B}_{0}$. The well known solution $[2,7]$ for the magnetic field $\mathbf{B}$ of helicon waves of angular frequency $\boldsymbol{\omega}$, azimuthal mode number $m$, and axial wave number $k$ in this plasma is

$$
\mathbf{B}=\mathbf{B}_{1}+\mathbf{B}_{2}
$$

where $\mathbf{B}_{\mathrm{j}}$ satisfies

$$
\nabla \times \mathbf{B}_{j}=\beta_{j} \mathbf{B}_{j}, \quad \mathrm{j}=1,2
$$

where 


$$
\beta_{1,2}=\frac{k}{2 \delta}
$$

and

$$
\begin{aligned}
& \delta \equiv \omega / \omega_{c} \\
& k_{w}^{2} \equiv \frac{\omega \omega_{p}^{2}}{\omega_{c} c^{2}}=\omega \frac{n_{0} e \mu_{o}}{B_{0}} .
\end{aligned}
$$

Here $\omega_{c}$ and $\omega_{\mathrm{p}}$ are the electron cyclotron and plasma frequencies, and we have adopted the notation of a more recent paper [9]. For a uniform plasma, the components of $\mathbf{B}$ can be written compactly in terms of Bessel functions:

$$
\begin{gathered}
B_{r_{j}}=A_{j}\left[\left(\beta_{j}+k\right) J_{m-1}\left(T_{j} r\right)+\left(\beta_{j}-k\right) J_{m+1}\left(T_{j} r\right)\right] e^{i(m \theta+k z-\omega t)} \\
B_{\theta_{j}}=i A_{j}\left[\left(\beta_{j}+k\right) J_{m-1}\left(T_{j} r\right)-\left(\beta_{j}-k\right) J_{m+1}\left(T_{j} r\right)\right] e^{i(m \theta+k z-\omega t)} \\
B_{z j}=-2 i A_{j} T_{j} J_{m}\left(T_{j} r\right) e^{i(m \theta+k z-\omega t)}, \quad j=1,2,
\end{gathered}
$$

where $A$ is an arbitrary amplitude and the transverse wavenumber $T$ is given by

$$
T_{j}^{2}=\beta_{j}^{2}-k^{2} .
$$

For the usual helicon wave, $\beta$ has the value $\beta_{1}$, the smaller of the two roots in Eq. (3). The larger root, $\beta_{2}$, describes a cyclotron wave which is important only at low magnetic fields $B_{0}$. For large $B_{0}$, above about $500 \mathrm{G}$, the cyclotron wave has such small radial wavelength that it is likely to be damped out within a short distance of the plasma surface. For simplicity, we consider, for the time being, the case $B_{0} \rightarrow \infty, \delta \rightarrow 0$, so that $\mathbf{B}_{2}$ can be neglected. We therefore take $\mathbf{B}=\mathbf{B}_{1}, \beta=\beta_{1}$, and $T=T_{1}$, suppressing the subscript 1 .

To form a plane-polarized wave near the axis of the discharge, we can linearly superpose waves of positive and negative $m$. Let these waves be denoted by $\mathbf{B}^{+}$and $\mathbf{B}^{-}$, with amplitudes $A^{+}$and $A^{-}$, respectively. For given $\omega_{c}, B_{0}, n_{0}$, and $k$, we see from Eqs. (3) and (4) that $\mathbf{B}^{+}$and $\mathbf{B}^{-}$have the same values of $\beta$ and $T$. The combined waves will then have the form

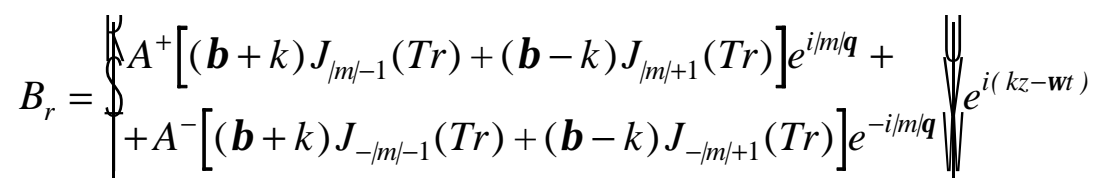

and similarly for $B_{\theta}$ and $B_{\mathrm{z}}$. Since the general case follows trivially, we can simplify the algebra now by specifying $m= \pm 1$. Suppressing the factor exp $i(k z-\omega t)$, we now have for the components of the total field

$$
\begin{aligned}
& B_{r}=A^{+}\left[\boldsymbol{\beta}+k \mathbf{g}_{\mathbf{0}}+\boldsymbol{\beta}-k \mathbf{g}\right] e^{i \theta}+A^{-}\left[\boldsymbol{\beta}+k \mathbf{g}_{2}+\boldsymbol{\beta}-k \mathbf{g}\right] e^{-i \theta} \\
& B_{\theta}=i A^{+}\left[\boldsymbol{\beta}_{+k} \mathbf{g}_{0}-\boldsymbol{B}_{-k} \boldsymbol{g}_{1}\right]^{i \theta}+i A^{-}\left[\boldsymbol{\beta}_{+k} \mathbf{g}_{2}-\boldsymbol{\beta}_{-k} \boldsymbol{g}_{1}\right] e^{-i \theta} \\
& B_{z}=-2 i T J_{1} \mathbf{d}^{+} e^{i \theta}-A^{-} e^{-i \theta} \mathbf{j}
\end{aligned}
$$


Here we have used the relation $J_{-\mathrm{m}}=(-1)^{\mathrm{m}} J_{\mathrm{m}}$, and the arguments of the Bessel functions $J_{\mathrm{m}}$ are understood to be $T r$. At $r=0, J_{2}$ vanishes and $J_{0}=1$. To form a plane-polarized field there in, say, the $x$ direction, we set

$$
A^{+} / A^{-}=\text {(- }-k \mathbf{g} \boldsymbol{\beta}+k \mathbf{g} R .
$$

Normalizing to $A^{+}(\beta+k)=1$, we see that Eq. (8) becomes

$$
\begin{aligned}
& B_{r}=\left[J_{0}+R J_{2}\right] e^{i \theta}+\left[J_{0}+R^{-1} J_{2}\right] e^{-i \theta} \\
& B_{\theta}=i\left[J_{0}-R J_{2}\right] e^{i \theta}+i\left[-J_{0}+R^{-1} J_{2}\right] e^{-i \theta} \\
& B_{z}=-2 i T \boldsymbol{\beta}_{+k} \mathbf{g} J_{1} \boldsymbol{d}^{\theta}-R^{-1} e^{-i \theta} \mathbf{|}
\end{aligned}
$$

The plane-polarized and circularly polarized components can be separated by adding and subtracting a term $\left(J_{0}+R J_{2}\right) \exp (-i \theta)$ in $B_{\mathrm{r}}$, and similarly in $B_{\theta}$ and $B_{\mathrm{z}}$. We then obtain

$$
\begin{aligned}
& B_{r}=\left(J_{0}+R J_{2}\right) 2 \cos \theta+J_{2}\left(R^{-1}-R\right) e^{-i \theta} \\
& B_{\theta}=-\left(J_{0}-R J_{2}\right) 2 \sin \theta+i J_{2}\left(R^{-1}-R\right) e^{-i \theta} \\
& B_{z}=2 T J_{1}(\beta+k)^{-1}\left[2 \sin \theta+i\left(R^{-1}-1\right) e^{-i \theta}\right]
\end{aligned}
$$

Using the definitions in Eqs. (6) and (9) and reinserting the final exponential factor, we can write the plane and circular components of Eq. (11) as follows:

$$
\begin{aligned}
& B_{r}=\left[J_{0}(T r)+R J_{2}(T r)\right] \cos \theta e^{i(k z-\omega t)} \\
& \text { Plane: } \quad B_{\theta}=-\left[J_{0}(T r)-R J_{2}(T r)\right] \sin \theta e^{i(k z-\omega t)} \\
& B_{z}=2 \sqrt{R} J_{1}(T r) \sin \theta e^{i(k z-\omega t)} \\
& B_{r}=2\left(\beta k / T^{2}\right) J_{2}(T r) e^{i(-\theta+k z-\omega t)} \\
& \text { Circular: } \quad B_{\theta}=2 i\left(\beta k / T^{2}\right) J_{2}(\operatorname{Tr}) e^{i(-\theta+k z-\omega t)} \\
& B_{z}=2 i(k / T) J_{1}(T r) e^{i(-\theta+k z-\omega t)}
\end{aligned}
$$

Here we have renormalized so that the plane-polarized component has unit amplitude on axis.

\section{COMPUTATIONS}

Before the field shapes can be computed, the possible values of $k-$ and, hence, of $\beta$ [Eq.(3)] and $T$ [Eq.(6)] — have to be determined. For a conducting cylinder, the boundary conditions are $E_{\theta}=0$ and $E_{\mathrm{z}}=0$. The latter is automatically satisfied if the conductivity is infinite, and then it follows from $\nabla \times \mathbf{E}=i \omega \mathbf{B}$ that $B_{\mathrm{r}}=0$ at $r=a$. From Eq. (10) we see that the condition $J_{0}+R J_{2}=0$ leads to eigenvalues of $k$ corresponding to different radial mades with $m=+1$. The condition $J_{0}+R^{-1} J_{2}=0$ leads to different values of $k$ for the $m=-1$ mode. Thus $B_{\mathrm{r}}$ cannot vanish at all $\theta$ for the same $k$ unless $|R|=1, k=0$. It is not possible to adjust the amplitude ratio $R$ to satisfy the boundary condition for the combined wave because $R$ has already been fixed by the condition of plane polarization on axis. For an insulating cylinder, 
the boundary condition in the helicon limit $(\delta \rightarrow 0)$ is $j_{\mathrm{r}}(a)=0$; and since $\nabla \times \mathbf{B}=$ $\mu_{0} \mathbf{j}$, we again require $B_{\mathrm{r}}(a)=0$, which is impossible. The problem is not resolved if we allow finite $\delta$ and the existence of the cyclotron wave $\mathbf{B}_{2}$. In that case, $E_{\mathrm{z}}$ no longer vanishes, even in the absence of dissipation, and the additional condition $E_{\mathrm{z}}(\mathrm{a})=0$ again leads to different eigenvalues of $k$ for the $m=+1$ and $m=-1$ modes. These values would give rise to a beat pattern as the combined wave propagates along $\mathbf{B}_{0}$. Fortunately, a recent study [9] has shown that, at high magnetic fields, the eigenvalues of $k$ for an insulating boundary are so closely spaced that the spectrum of possible $k$ 's is almost continuous. This is because the amplitude of the external wave in vacuum can adjust itself to satisfy the boundary condition. Therefore, we may pick an eigenvalue of $k$ for the $m=+1$ mode, say, and assume that an $m=-1$ mode with nearly the same value of $k$ will exist. The difference will be so small that the beat pattern will not be detectable within the length of the apparatus. These considerations apply only to the ideal case. When damping and plasma nonuniformity are considered, the $k$ spectra will be broadened anyway.

For illustrative purposes we have chosen the following parameters: $B_{0}=800 \mathrm{G}, n_{0}=$ $10^{13} \mathrm{~cm}^{-3}, a=5 \mathrm{~cm}, f=13.56 \mathrm{MHz}$. The boundary condition $B_{\mathrm{r}}^{+}(a)=0$ then leads to $k=$ $0.336 \mathrm{~cm}^{-1}\left(18.7 \mathrm{~cm}\right.$ wavelength) for the $m=+1$ component. The condition $B_{\mathrm{r}}^{-}(a)=0$ would give $k=0.226$ (27.8 cm wavelength) for the $m=-1$ component. The beat wavelength for these two modes would be $57 \mathrm{~cm}$. However, we would expect that the antenna's $k$-spectrum would dictate $k$-values which are much closer together than these, and that the dominant $k$ value would be that of the dominant $m=+1$ mode. The following graphs were therefore computed for $k=0.336 \mathrm{~cm}^{-1}$.

Fig. 1 is the $B$-field pattern in a transverse plane for the plane-polarized component, given by Eq. (12). This pattern is stationary in the laboratory frame. Fig. 2 shows the instantaneous $B$-field pattern for the circularly polarized component, given by Eq. (13). This pattern is left-hand polarized and rotates counterclockwise as viewed along $\mathbf{B}_{0}$. This can be seen from the exponential factor in Eq. (13). On the other hand, at any given position $(r, \theta)$, the field lines rotate clockwise as a function of time. This can be seen by forming the linear combinations $B^{\mathrm{R}}=\left(B_{\mathrm{r}}-i B_{\theta}\right) / \sqrt{ } 2$ and $B^{\mathrm{L}}=\left(B_{\mathrm{r}}+i B_{\theta}\right) / \sqrt{ } 2$, which represent the right- and left-hand polarized components of the vector $\mathbf{B}$. From Eq. (13) we see that $B^{\mathrm{L}}=0$.

Eqs. (12) and (13) show that the plane-polarized component has maximum amplitude on axis whereas the circularly polarized component vanishes there. As $r$ increases, the circular component becomes more important and it dominates at the edge. This is shown in Fig. 3, where the time-averaged field $\langle|\mathbf{B}|\rangle$ is plotted against radius. The plane-polarized component has an average amplitude that depends on angle, while that of the rotating component, of course, does not.

From Faraday's Law one easily finds that the electric and magnetic field lines are perpendicular to each other in a transverse plane. The electric field pattern corresponding to Fig. 1 is shown in Fig. 4. The ionization produced by this $m= \pm 1$ mode may be azimuthally asymmetric because the average energy deposition by the plane-polarized component will depend on $\theta$, while, of course, that of the circular component will not. To compute this, we note that the energy loss $W$ is proportional to $\eta j_{z}^{2}$, where $\eta$ is an effective resistivity [8], and $j_{z}$ is essentially $\left(\beta / \mu_{0}\right) B_{\mathrm{z}}$, as shown earlier. Hence, $W$ is proportional to $B_{\mathrm{z}}{ }^{2}$. We are interested in the asymmetric part of this, which is given by Eq. (12) for the plane-polarized component. We see that $W$ is maximum at $\theta=\pi / 2$ and $\operatorname{Tr}=1.841$, where $J_{1}(\operatorname{Tr})$ has its first maximum. 
The contours of $W / W_{\max }=0.9$ and 0.5 are shown in Fig. 4, superposed on the $E$-field pattern. If the $E$-field is excited by a Nagoya Type III antenna, we would expect the straight legs of that antenna to be at $\theta= \pm \pi / 2$. The asymmetric part of the energy deposition would, therefore, be aligned with the parallel legs of the antenna. This contradicts the orientation reported by Kim et al. [6], which is at $90^{\circ}$ to this. If that observation is confirmed, it would be difficult to explain by any theory.

Kim et al. [6] have pointed out that quadrupolar antennas could excite $m= \pm 2$ patterns, and indeed have reported seeing them. The theoretical patterns for plane-polarized $m$ $= \pm 2$ modes can be found from the general equations (5) and (7). The result is

$$
\begin{aligned}
& B_{r}=\left[J_{1}(T r)+R J_{3}(T r)\right] \cos 2 \theta e^{i(k z-\omega t)} \\
& \text { Plane: } \quad B_{\theta}=-\left[J_{1}(T r)-R J_{3}(\operatorname{Tr})\right] \sin 2 \theta e^{i(k z-\omega t)} \\
& B_{z}=2 \sqrt{R} J_{2}(T r) \sin 2 \theta e^{i(k z-\omega t)} \\
& B_{r}=2\left(\beta k / T^{2}\right) J_{3}(T r) e^{i(-2 \theta+k z-\omega t)} \\
& \text { Circular: } \quad B_{\theta}=2 i\left(\beta k / T^{2}\right) J_{3}(T r) e^{i(-2 \theta+k z-\omega t)} \\
& B_{z}=-2 i(k / T) J_{2}(T r) e^{i(-2 \theta+k z-\omega t)}
\end{aligned}
$$

The magnetic and electric field patterns of Eq. (14) are shown in Figs. 5 and 6.

\section{CONCLUSION}

We have shown that a plane-polarized helicon wave is in principle possible in a magnetized cylinder uniformly filled with plasma. This wave must be accompanied by a left-hand circularly polarized wave, which is of comparable magnitude at large radii but is small relative to the plane-polarized component near the axis. To satisfy conducting boundary conditions, the right- and left-hand rotating components of the plane-polarized wave will have different axial wavelengths, giving rise to a beating phenomenon. An insulating boundary, however, will permit both components to have nearly identical wavelengths. These conclusions hold even if the helicon waves are significantly coupled to electron cyclotron waves, as would occur at weak magnetic fields. A small amount of damping would broaden the $k$-spectra enough to allow both components to have the same wavelength. Plane-polarized waves can also exist in a nonuniform plasma, although the field patterns would not then be expressible in terms of Bessel functions but must be computed using another formulation [10].

If plane-polarized helicons are generated by a straight antenna of $m=1$ symmetry, such as a Nagoya Type III antenna, one would expect the asymmetric part of the energy deposition to be aligned with the parallel legs of the antenna. In practice, it may be difficult to excite the $m=+1$ and $m=-1$ components with comparable amplitudes because antenna coupling computations [11] show that the $m=+1$ mode is much more strongly excited than the $m$ $=-1$ mode if the density profile is peaked on axis. Furthermore, it has been found that the $m=$ -1 mode is damped faster than the $m=+1$ mode as it propagates along $\mathbf{B}_{0}$ [12]. In view of 
these practical considerations, it is surprising that plane-polarized modes, though theoretically possible, have been seen [6].

\section{ACKNOWLEDGMENTS}

This work was supported by the National Science Foundation, the Wisconsin Engineering Research Center for Plasma-Aided Manufacturing, and the Semiconductor Research Corp. We thank John Evans for help with the algebra and Donald Arnush and David Blackwell for their comments and communication of preliminary results.

\section{REFERENCES}

[1] R.W. Boswell, Plasma Phys. Control. Fusion 26, 1147 (1984).

[2] J.P. Klozenberg, B. McNamara, and P.C. Thonemann, J.Fluid Mech. 21, 545 (1965).

[3] F.F. Chen, Phys. Plasmas 3, 1783 (1996).

[4] T. Watari, T. Hatori, R. Kumazawa, S. Hidekuma, T. Aoki, T. Kawamoto, M. Inutake, S. Hiroe, A. Nishizawa, K. Adati, T. Sato, T. Watanabe, H. Obayashi, and K. Takayama, Phys. Fluids 21, 2076 (1978).

[5] F.F. Chen, "Plane-polarized whistler waves", Bull. Amer. Phys. Soc. 35,. 2101 (1990).

[6] J.H. Kim, S.M. Yun, and H.Y. Chang, IEEE Trans. Plasma Sci. 24, 1364 (1996).

[7] A.R. Ellingboe, and R.W. Boswell, Phys. Plasmas 3, 2797 (1996).

[8] F.F. Chen, Plasma Phys. Control. Fusion 33, 339 (1991).

[9] F.F. Chen and D. Arnush, "Generalized theory of helicon waves I: Normal modes", UCLA PPG-1562 (Aug 1996), submitted to Phys. Plasmas.

[10] F.F. Chen, M.J. Hsieh, and M. Light, Plasma Sources Sci. Technol. 3, 49 (1994).

[11] D. Arnush and F.F. Chen, "Damping of low-field helicon-TG modes", Bull. Amer. Phys. Soc. 41, 1535 (1996).

[12] D.D. Blackwell and F.F. Chen, "Plane-polarized modes in a helicon discharge", Bull. Amer. Phys. Soc. 41, 1535 (1996). 


\section{FIGURE CAPTIONS}

1. Pattern of magnetic field lines for the plane-polarized component of a helicon wave comprising superposed $m=+1$ and $m=-1$ azimuthal modes. Assumed parameters: $800 \mathrm{G}$, $10^{13} \mathrm{~cm}^{-3}, 13.56 \mathrm{MHz}, 5 \mathrm{~cm}$ radius. This pattern is fixed with respect to the antenna.

2. Same as Fig. 1, but for the circularly polarized component. This pattern rotates in the clockwise direction if $\mathbf{B}_{0}$ is out of the plane of the page. The $B_{\mathrm{z}}$ component of the field is not shown, and the line spacing is not indicative of the field strength.

3. Relative time-averaged amplitudes of the plane and circularly polarized components.

4. Electric field pattern corresponding to the mode shown in Fig. 1. The broad lines are contours of constant energy deposition (at $90 \%$ and $50 \%$ of maximum) for the planepolarized component.

5. Magnetic field pattern for a plane-polarized $m=2$ wave.

6. Electric field pattern for a plane-polarized $m=2$ wave. 


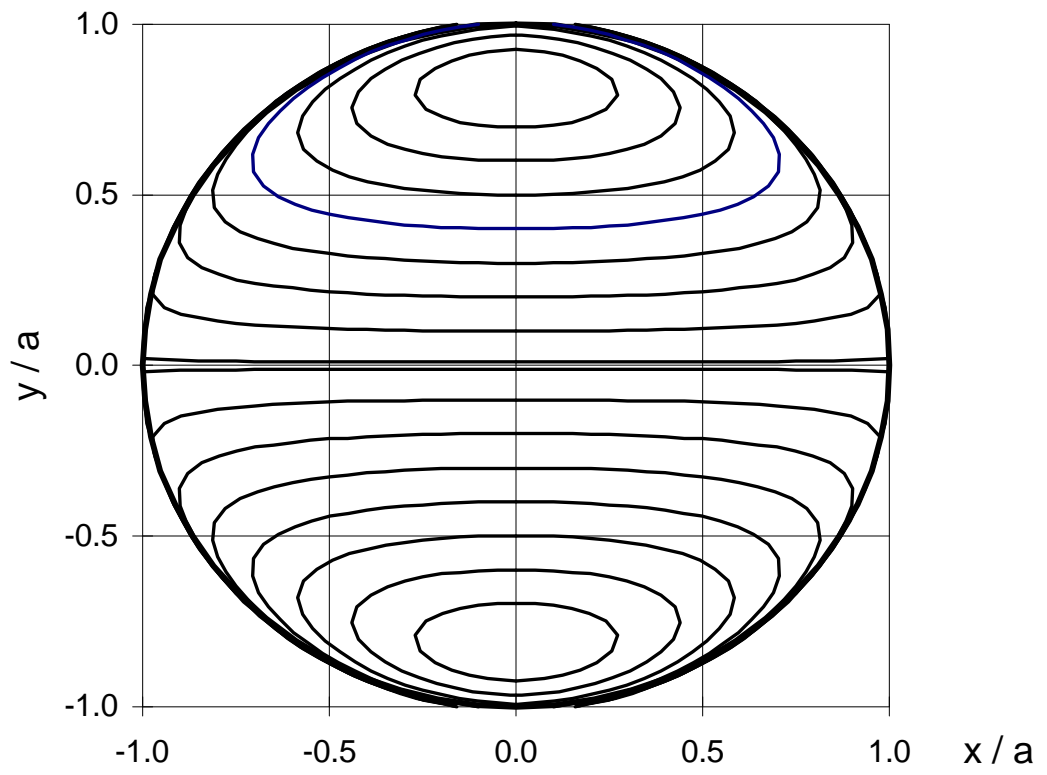

Fig. 1

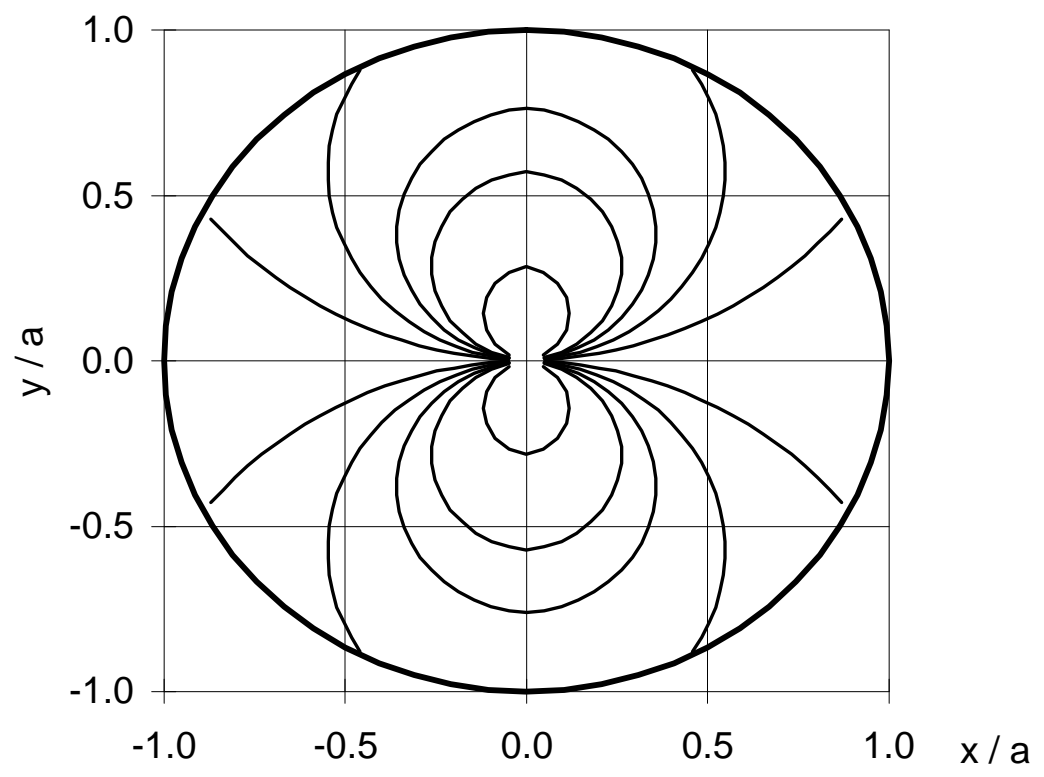

Fig. 2 


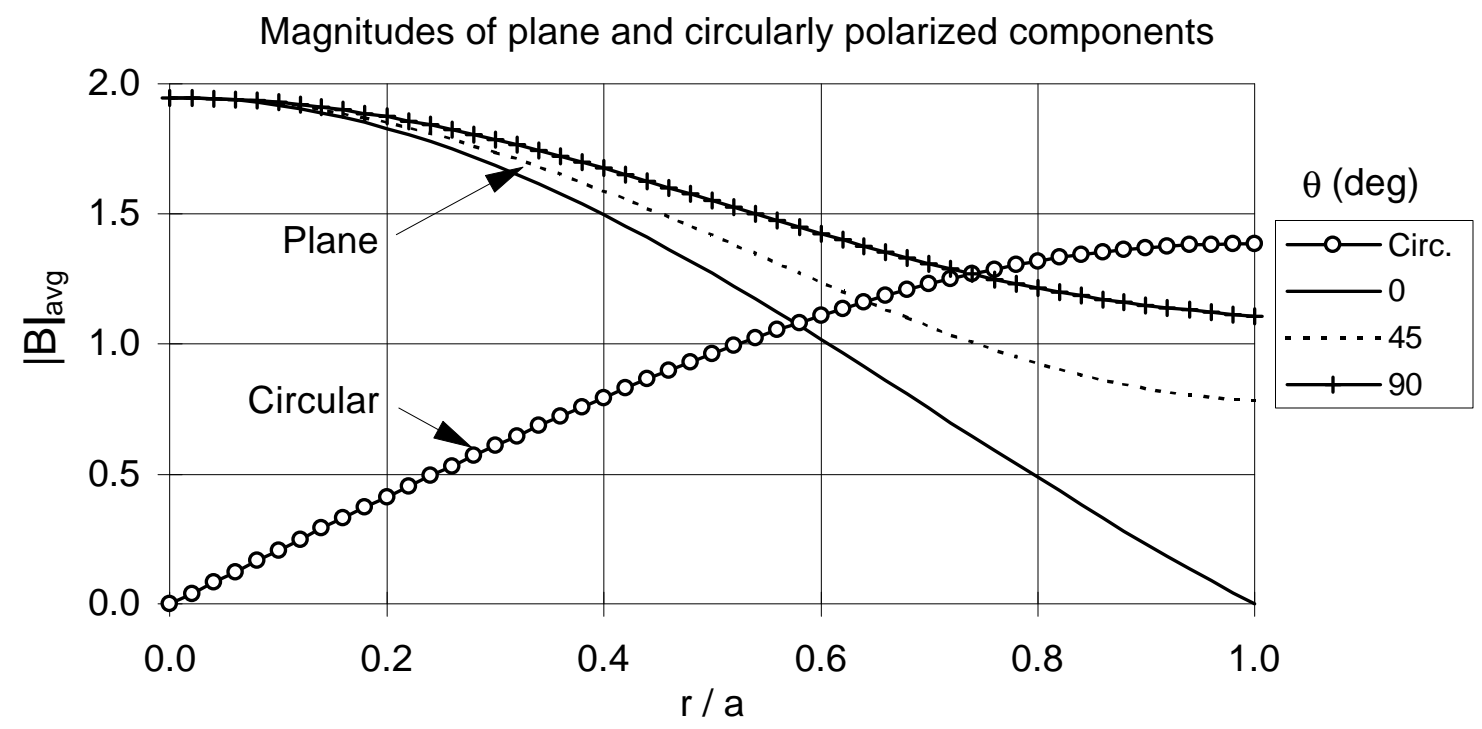

Fig. 3

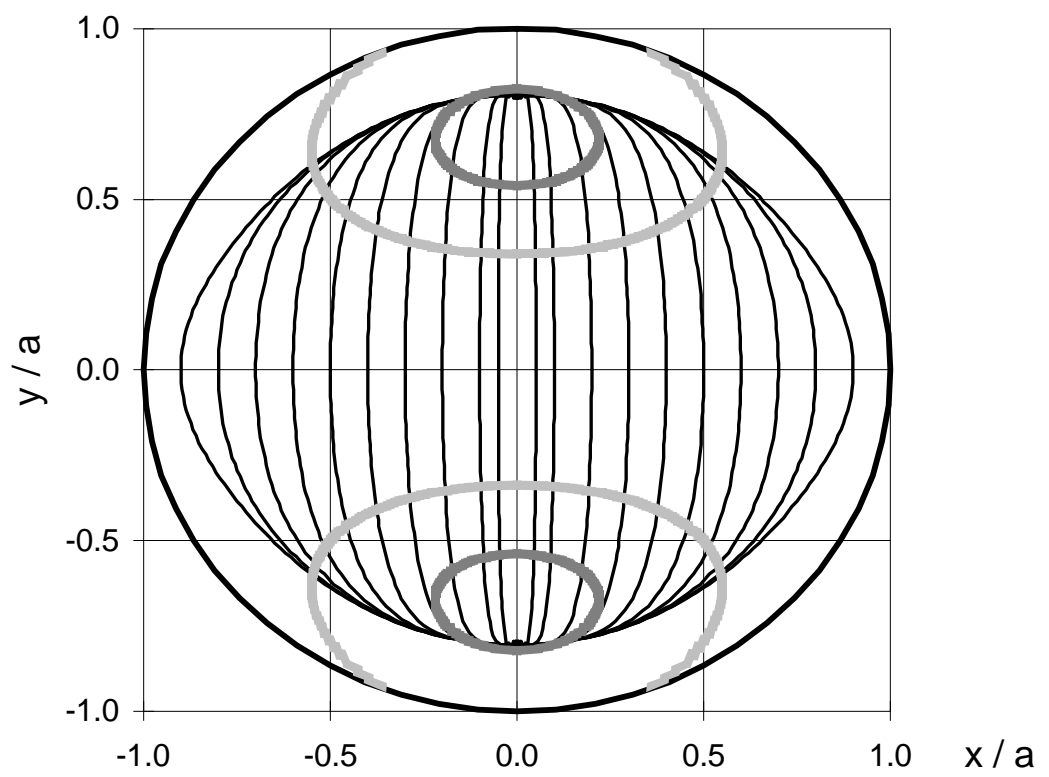

Fig. 4 


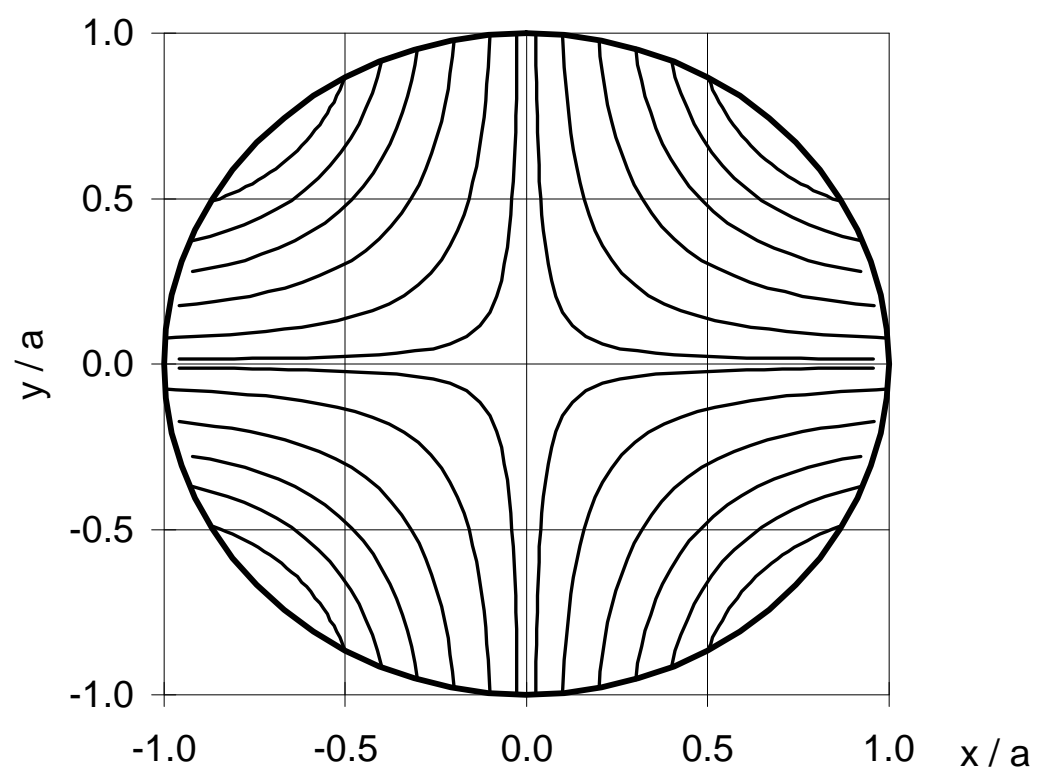

Fig. 5

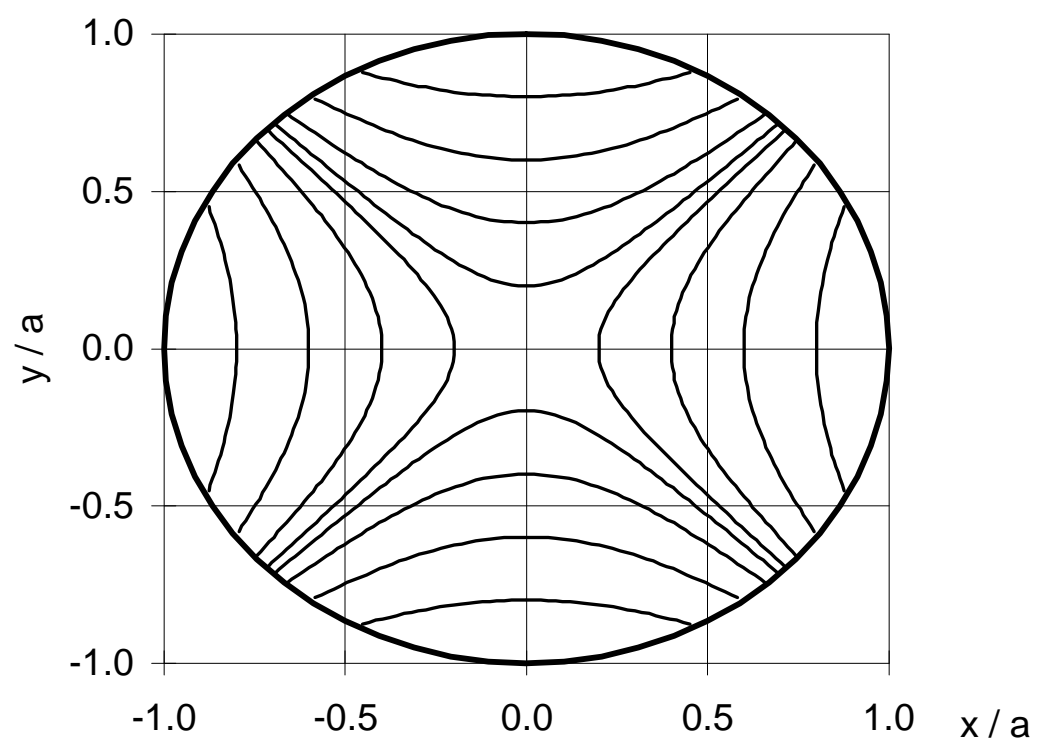

Fig. 6 